\title{
The Perception of Pathumthani Residents toward its Environmental Quality, Suburban Area of Thailand
}

\author{
Pawinee lamtrakul $\mathrm{A}^{*}$, Sararad Chayphong ${ }^{\mathrm{A}}$ \\ Received: January 20, 2021 | Revised: June 20, 2021 | Accepted: June 20, 2021 \\ doi: 10.5937/gp25-30436
}

\begin{abstract}
The urbanization process is often concentrated mostly in urban areas, resulting into urban development sprawl. This has effects on the lifestyles and activities of urban people, which in turn significantly affects the health of the city. The quality of the urban environment plays an important role in public health with respect to urban issues ranging from quality of utilities and services to quality of life. This study aims to study the perception of Pathumthani residents toward its environmental quality through spatial cluster analysis. A total of 1,000 sets of data collected from the interview survey among residents or commuters traveling through Pathumthani province was used for this study. The residents' response towards environmental factors was examined through the classification of their different opinions among built environment and health aspects. The statistical analysis which was performed in this study was cluster analysis to demonstrate its relationship. With the level of satisfaction on environment aspect and health status in indicating number of congenital diseases, the result of this study found that condition of living environment (through Likert scale) affects the urban health with statistical significance of $(P<0.05)$. Therefore, provincial health policy should focus more on developing a healthy city in consistence with economic and social development while putting adequate mechanisms for environmental surveillance monitoring at the community level. The result of study can confirm the usefulness of this unconventional approach by asking residents or commuters about their satisfaction on built environment which can represent as an evidencebased planning approach by linking local people attitudes and translating them into creating liveable and better urban environmental quality. To have a good understanding of local people preferences, the recommendation to be given to the capacity of communities can be focused for improving people's quality of life by providing better accessibility, high quality of infrastructures and services. Finally, a set of features of satisfied built environment can help to support the continued growth of the city in term of basic need and sufficiency provision of facility and utility system.
\end{abstract}

Keywords: built environmental; health; satisfaction; quality of life; urbanization

\section{Introduction}

The urbanization process in the developing world has been occurring in a rapid pace with high growth concentration mostly in urban areas (World urbanization prospects, 2018). The quality of the urban en- vironment has become an essential element to be considered in public health discourse like issues ranging from solid waste disposal, safe water and sanitation as well as safety and injury prevention. Health is de-

\footnotetext{
A Director, Center of Excellence in Urban Mobility Research and Innovation, Faculty of Architecture and Planning, Thammasat University, Pathumthani, 12120, Thailand; iamtrakul@gmail.com; s_ararad@hotmail.com

* Corresponding author: Pawinee lamtrakul; e-mail: iamtrakul@gmail.com
} 
fined as a state of complete physical and mental which represent not just a lack of disease or weakness, but also includes a social well-being. To reach the highest standards of health is an ultimate goal in achieving and exemplifying one of the fundamental rights of all human beings irrespective of race, religion, political beliefs, economy or society (WHO, 1946). For Thailand, the direction of health movement has changed over time, originally focusing on AIDS prevention and control, development and improvement of occupational health work, environmental health work, expansion of quality of public service, consumer protection, child health development, agricultural development, and traffic pollution problems solution (Development Plan No. 1-7: 1961-1996). This led to a development strategy that focuses on the development of the "people" as a whole and effective management to restore the economy. It is imperative to note that health is a well-being that consists of complete and balanced physical, mental, social and spiritual well-being a person. The development of public health is carried out in relation to various economic, social, cultural, values, behavior and environmental factors. The health policy is planned to comprehend the problems more holistically (Development Plan No. 8-9: 1997-2006) and immediately after the $10^{\text {th }}$ National Development Plan, balancing and sustainability became the center of emphasis. Balancing the developmental strategy of the country is achieved by linking all dimensions of integrated and holistic development including the "human, social, economic, environmental and political dimensions". Consequently, it can be seen that the issues to be considered in an urban health is not just the health factor, but other influencing factors should also be included.

However, in this current age of chronic diseases, association between population health and the built environment still remained. It can be seen that physical spaces can directly expose people to urban pollutants and influence on people lifestyles (Perdue et al., 2003). The consideration of the built environment includes all the human-made physical spaces which corresponds to the spaces for living, recreation and working. The physical structures like urban components were engineered and designed by people which became the places for our everyday life of working, living, playing and socialization. Also, urban physical is affecting to the enhancement of social capital in either positive or negative perspectives (Iamtrakul et al., 2018). These urban elements include our buildings, furnishings, public open spaces, roads, utilities, and other infrastructures which affects our health condition through both direct and indirect pollutions and externalities (Collaborative on health and the environment, 2016). The built environmental factors can generate both positive and negative impact on the urban health depending on an appropriateness of the planning and development. Its positive effect to the cities can be viewed from composition of green spaces, sidewalks and bicycle lanes that promotes physical exercise and mental health, improved markers of cardiovascular and metabolic health (Centers for Disease Control and Prevention, 2011; Office of the Surgeon General, 2015). In terms of the negatives, it contains built environment factors associated with more motor vehicle-pedestrian incidents. These increasing exposures consists of harmful contaminants in urban ambience which can reduce life expectancy and worsen the ill effects of some respiratory conditions (Teo et al., 2015; Grant et al., 2009). Therefore, the level of carbon consumption in our environments must be reduced since carbon has been proven to be impactful on human conditions and behaviors in a variety of ways (Ebmeier, 2012). The built environment also includes housing component and several housing factors are associated with mental and physical health impacts, e.g., air quality, infestation, noise, lighting, housing tenure and design (Macdonald, and Thompson, 2003). In the case of Thailand, one of the countries that has experienced significant changes in various areas of urban growth showed the main reason for rapid expansion of the urban areas due to peoples' migration to bigger cities. It is projected that in near future, more than 73 percent of the Thai population will become urban (Office of knowledge management and development, 2015). Although the greatest growth of population has occurred in Bangkok and vicinity (5 province namely; Nakhon Pathom, Pathumthani, Nonthaburi, Samut Prakan, Samut Sakhon), the unfortunate situation is that it has also created environmental problems such as dispersion of environmental pollution caused by emissions of pollutants from vehicles, industrial suburbs and houses, as well as pressures in demand for utilities. These challenges also include lack adequate of drinking water, waste and hazardous waste from homes, hospitals and industries, congested traffic caused by the rapid increase in number of vehicles etc. (Bureau of technical advisors, 2020).

In terms of social and economic problems, these are obviously seen in the outskirt are of Bangkok due to various activities concentrated disproportionately within this zone. A complex mix of activities around the industrial spaces, living neighborhoods, and commercial areas has resulted in the diversity of the population within the area. Considering the urban sprawling phenomena, the suburban areas also induce a large concentration of commercial activities, educational institutions as well as industrial estates and the regional market that attracts visitors from neigh- 
boring provinces and represented as regional attraction nodes. A wide range of activities and social diversity can reflect the demand on city's infrastructure both availability and quality of services which are at the core of many of the challenges faced by rapidly urbanization.

According to the Pathumthani Provincial Health Office (2020), there are 6 major public health problem areas in hierarchical order of importance which consist of: 1) non-communicable diseases (NCDs), disease group include mental health/ drugs/traffic accidents, 2) communicable diseases, spread by insects, 3 ) environment related illnesses such as exposure to general waste/solid waste/waste water/unhygienic food/ pesticide residues, 4) dependency (i.e., home addiction) bedridden/falls/dementia, 5) mother and child health and 6) elderly health. Therefore, urban development should be seen as an essential part of driving force for an urban growth capable of creating a wide range of urban utilization activities. However, in situation where activity within the city is overly developed, it could lead to problem dimensions that can be impactful on the health of the citizens of the city; leading to deteriorating state or the risk within the city. Therefore, this study focused on the study of the relationship of the built environmental and urban health based on residents' perspectives in order to provide guidance in urban health development as regards development issues in the urban environment. This study is expected to generate a positive influence and a fundamental human need in order to live with complete happiness under urban development.

\section{Data and methods}

\section{Literature review}

When we look at the components of the built environment, it is expected it involves several material determinants of health including housing, neighborhood conditions and transport routes because they have an effect on the social, economic and environmental conditions (Barton et al., 2006). Good health impact is directly or indirectly dependent on the built environment in terms of health condition and wellbeing; including those traditionally associated with infrastructure planning and environmental health. These externalities include air quality (indoor and outdoor), climate, water quantity and quality, noise, traffic-related injuries, etc. (Borasi, et al., 2012; Frumkin et al., 2004; Sustainable development commission, 2008). The quality of space utilization is obviously depending on living and working physical condition which includes buildings, streets, and built environment components. Consequently, the health of individuals and populations is affected by the surrounding environment that is planned and managed by urban development. This process is an essential part that controls and drives urban growth through which a wide range of urban activities are created. However, there are series of activities that can cause physical and environmental degradation in service areas capable of affecting the society and causing economy disorder. It has become obvious that all these impacts on the urban health ranging from the deteriorating conditions to the risks within the city has caused a shift in conceptualizations of health and disease prevention. The paradigm shift is from the treatment of illness in individuals to disease prevention and health promotion in populations, with more focus on the impact of the environment on collective well-being (McLeroy et al., 1988; Stokols, 1996).
The built environment results in the differences between the objective environment and subjective perception of the environment through residents' view. Perceptions of different people may influence the understanding of these cognitive representations, and perceptions of the environment which may not correspond to objective reality (Ewing \& Handy 2009). Perceptions of the built environment reflect an individual's interaction with the actual environment, involving an awareness and perception through their primary receptive senses (Sherrington, 1961). Consequently, respondents' direct experiences with specific residential location can be captured and reflect through their perspective which plays an important role in disease dynamics and in determining the health of individuals, particularly from the built environment (Pinter-Wollman et al., 2018). The current understanding of health combines absence of disease and a state of complete physical, mental and social well-being which emphasize the approach of prevention as important as cure to be explored for long-term solutions (Tsekleved \& Cooper, 2017).

Therefore, the definition of health to be considered in the built environment design should cover a state of complete physical, mental, and social well-being and not merely the absence of disease or infirmity (WHO, 1946). However, there is relationship between health outcomes and risk factors associated with the built environment like physical activity, dietary intake, blood pressure, obesity, cardiovascular disease, diabetes mellitus, cardiometabolic syndrome, and mental health (Nathan et al., 2018; Durand et al., 2016; Christian et al., 2011; Sundquist et al., 2015). On a final note, the built environment has been increasingly recognized as being associated with health out- 
comes which has been regarded as part of the problem, though it differed among diverse groups, ranging in different socio-economic characteristics. However, all concerns factor that are based on individual level associated with built environment must be included as a part of the solution (Villanueva et al., 2013). Different residents to perceive the same physical space through different lens can be evidence supporting the importance of environmental variables in health and perception in environmental quality which is a key decision-point for planners to understand prior to any design, planning and policy intervention.

\section{Study area and data collection}

Data for this study was collected from a data sample using the method for calculation of population size of Taro Yamane's calculation formula (Taro, 1967). It was considered with the acceptable size error of 95\% from the population of 1.129 million people (2017). For a wider coverage and a larger data sample size, a total of 1,000 data sets were collected from people living within the area or traveling through Pathumthani Province which is the representative of suburban area of Thailand (Figure 1).

The study area consists of 7 districts which are; 1 ) Nong Suea District, 2) Thanyaburi District, 3) Lam Luk Ka District, 4) Khlong Luang District, 5) Lat Lum Kaeo District, 6) Sam Khok District, and 7) Mueang Pathumthani District. The number of questionnaires which were distributed in the study area were determined from the population size of each district to demonstrate the appropriate representation of the population in each area. All districts were achieved through the number of distributions of questionnaires to cover the number of samples in spatial areas of all grids.

\section{Procedure and analysis}

This study focused on the relationship of built environment and urban health by perform the evaluation through cluster analysis as depicted in Figure 2. A total set of 1,000 data from residents living within the area or traveling through Pathumthani province was input into the step of analysis which consisted of 2 group of variables as follows:

1. Built environment factors: comprises of water, air quality, dust pollution, noise, industrial area, housing, infrastructure, transportation. These factors were assessed by using Likert scale to demonstrate level of problem about built environment factor. The level of assessment can be classified by Likert scale ranging from 1 [the lowest satisfaction of environment aspect] to 5 [the highest satisfaction of environment aspect] in all attributes of built environment factors.

2. Health status factors: comprises of congenital disease and it was explained by variable of health status in indicating number of chronic diseases. The data of congenital disease were collected from questionnaires by asking whether there is an underlying disease or not. The level of health was classified in 5 level which 1 score represents the lowest level of

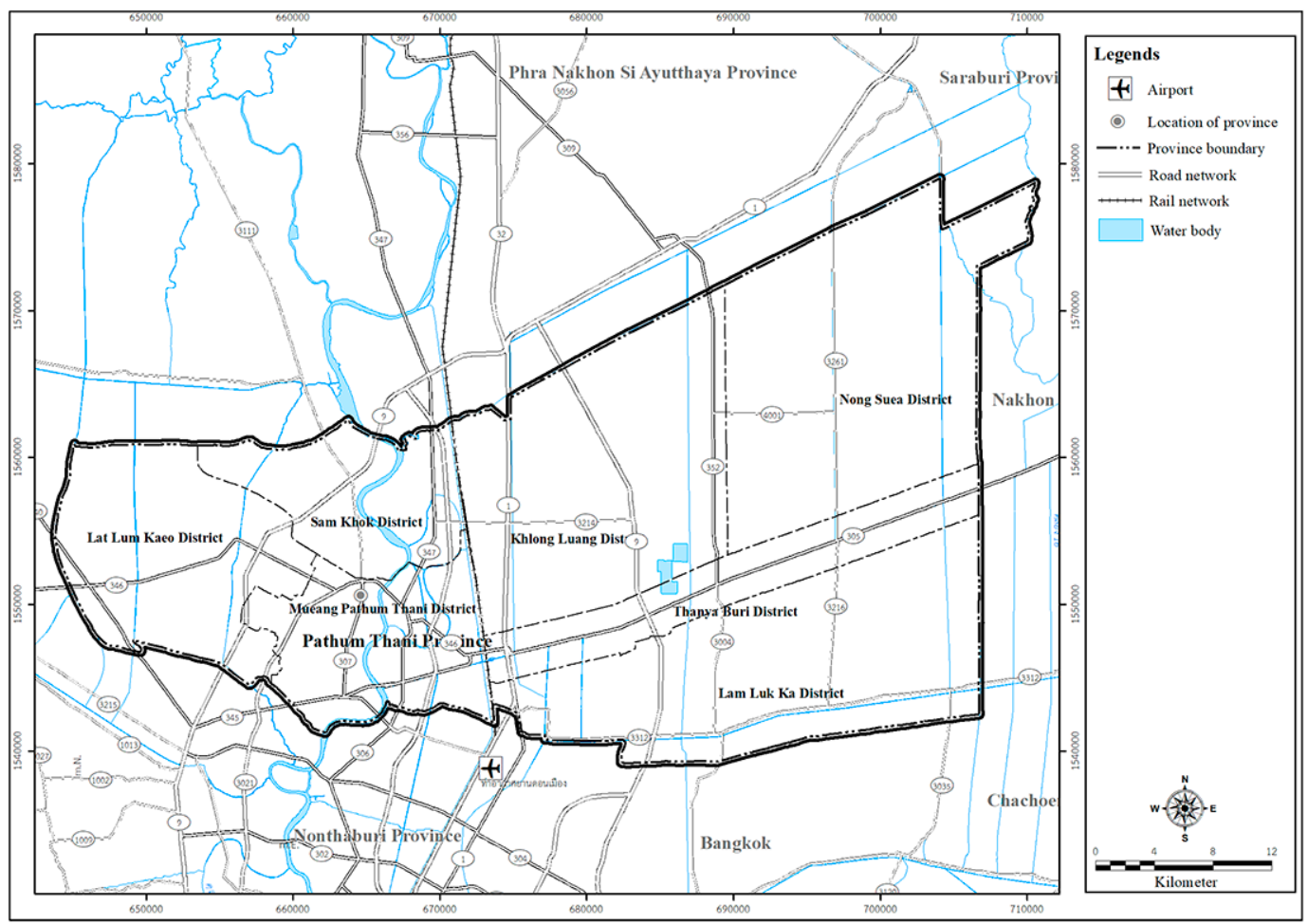

Figure 1. Study area: Pathumthani province 


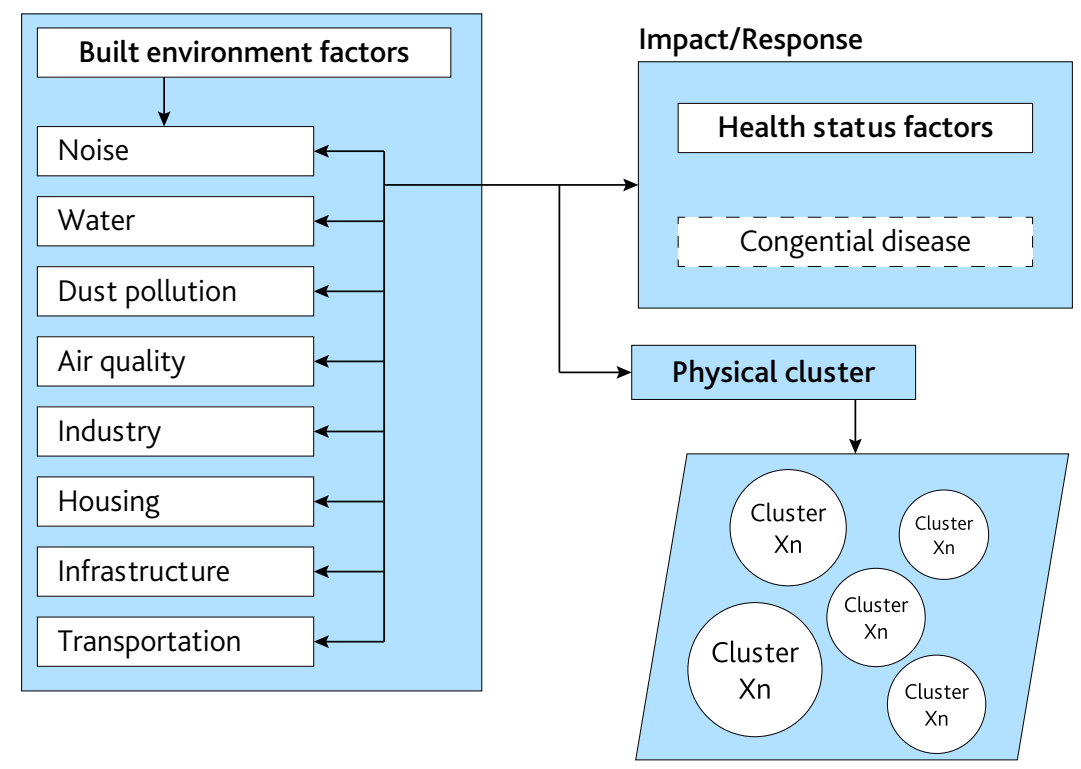

Figure 2. Framework of study

health status (respondents with more than 4 diseases) and 5 score demonstrates good health condition (respondent without any congenital disease).

A cluster analysis was performed to classify different built environment factors in the study area and its relationship with health condition of residents. To evaluate all aspect of the built environment factors that affects the health status of samples from residents in the study area, the level of significance for the analyses was set at $\mathrm{p}<.05$. Thus, the imported variables to be input in the clustering analysis consist of the set of data from built environment factors and health status factors which was explained in the previous details.

\section{Results}

This study was conducted to determine the relationship between the factors affecting the development of healthy cities. The result of analysis was considered through a spatial visualization by using geographic information system (GIS) to classify different 'the built environment' factors in the study area. The relationship of built environment that affects the health condition by using a cluster analysis. The details of the study results are as follows:

\section{Built environment}

The factors that are considered for environmental quality assessment by residents' perspective in built environments includes water, air quality, dust pollution, noise, industrial area, housing, infrastructure and transportation. These factors were assessed by using Likert scale range from 1 to 5 score in all built environment factors. The result of analysis by using average statistics were visually demonstrated in Figure 3 and Figure 4.

Most problems in the built environment were found in infrastructures $(\bar{x}=2.63)$, dust pollution $(\bar{x}=2.67)$, housing $(\overline{\mathrm{x}}=2.68)$, air quality $(\overline{\mathrm{x}}=2.73)$, transportation $(\bar{x}=2.84)$, noise pollution $(\bar{x}=2.85)$, water quality $(\bar{x}=2.97)$ and industrial area $(\bar{x}=3.06)$, respectively. In overall, it was discovered that the direction of opinions and attitudes of the respondents reflect the current situation of urban development and health characteristics. By considering air quality and noise level, it was found that the main sources are from an industrial area which has been influenced by rapid growing of capital city, Bangkok. The installation of air quality monitoring station around the study area provides an information of pollution source which are mainly from factories and traffic congestions. Moreover, recent development trend of suburban area has induced urban activities through urbanization, industrialization, which also increase in dust pollution problems. Additionally, it was found that majority of citizens lived in residential areas with air pollution exceeds the safe level reported by the Pollution Control Department. A non-negligible driver of rapid growth of suburbanization resulting in huge demand of water supply due to economic expansion and more coverage of transport network. Moreover, housing projects and industrial employment area has been constantly evolving which created impact on increasingly serious water environment problems of approximately 60 percent of the agricultural area of the province. 


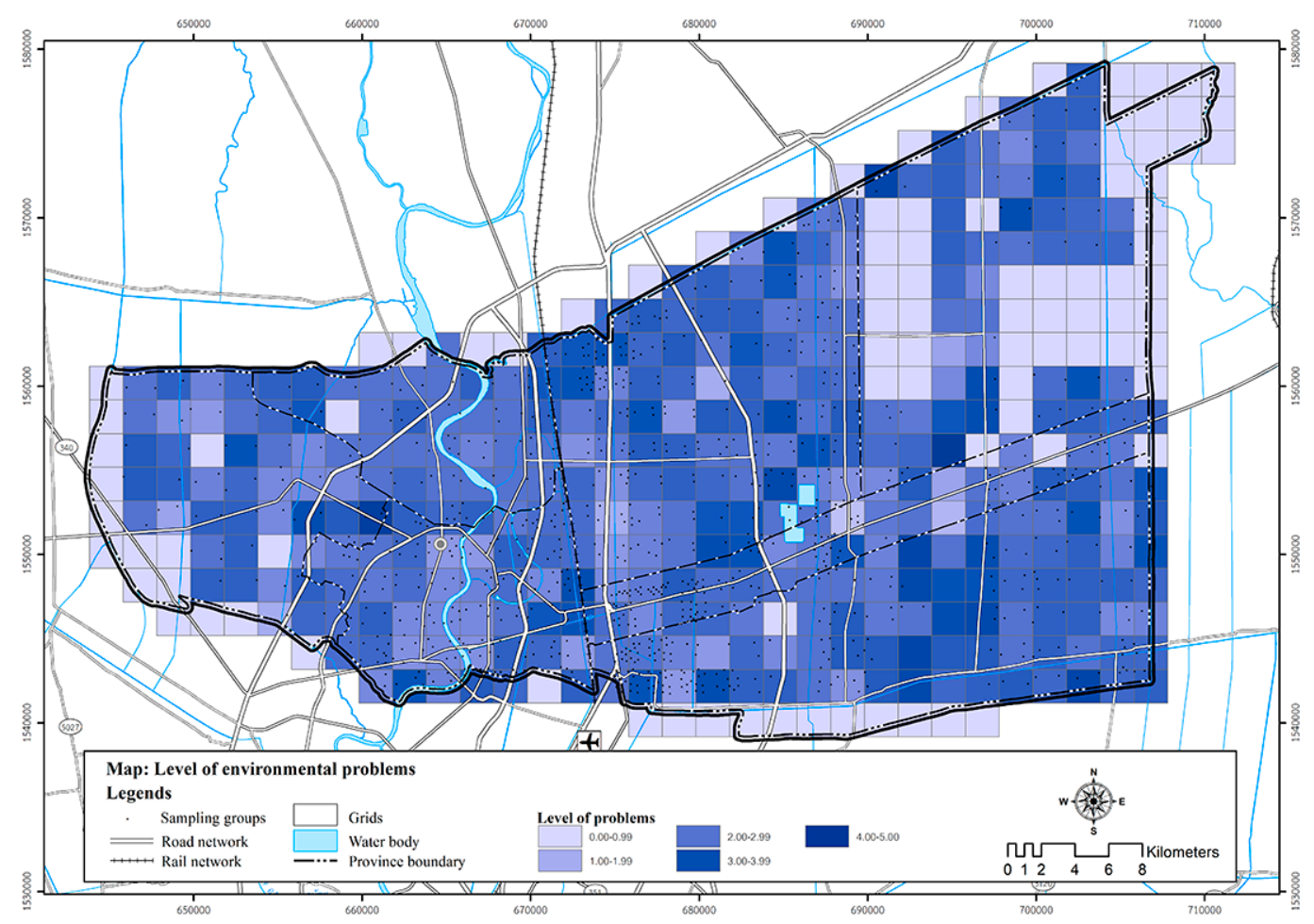

Figure 3. Summary of average score of overall built environment classified by grids

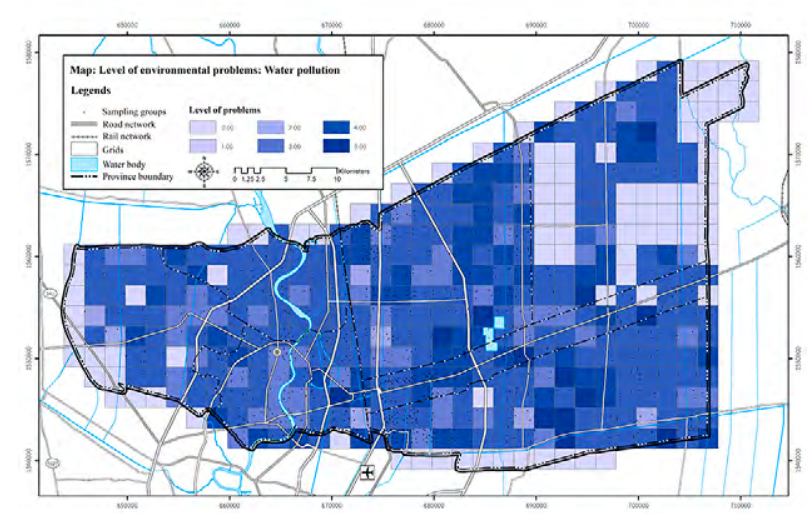

A. Water pollution

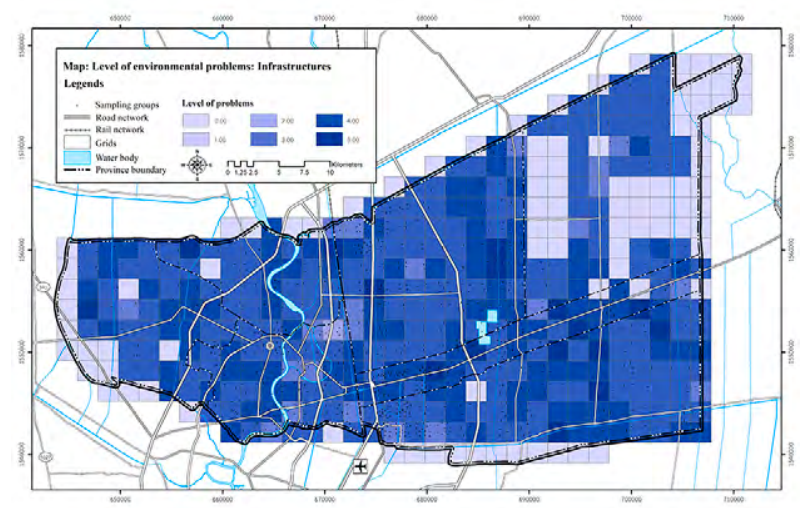

C. Infrastructures

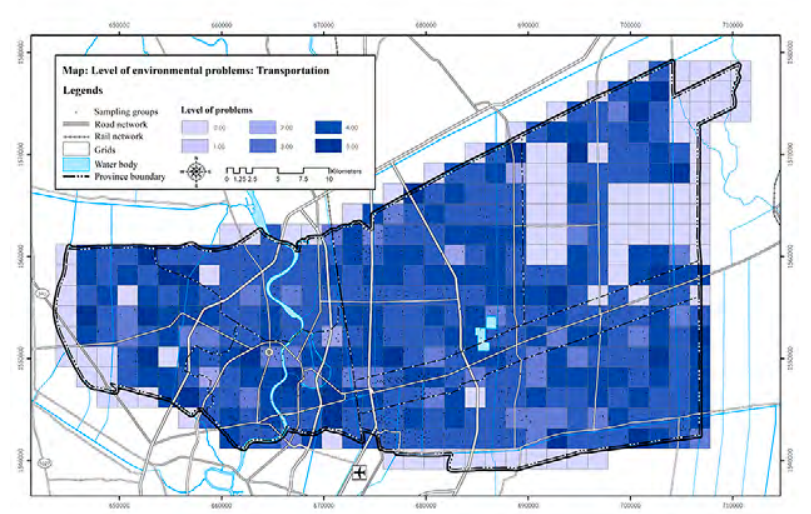

B. Transportation

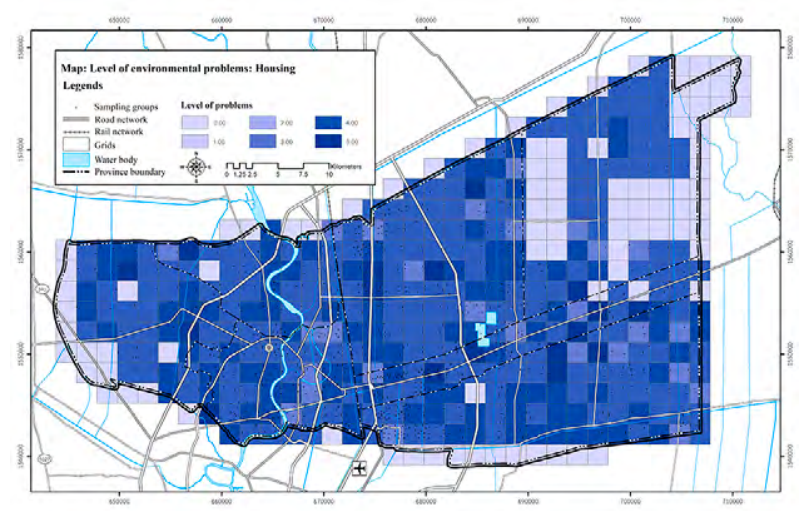

D. Housing

Figure 4. Average score of different built environment factor (continued on next page) 


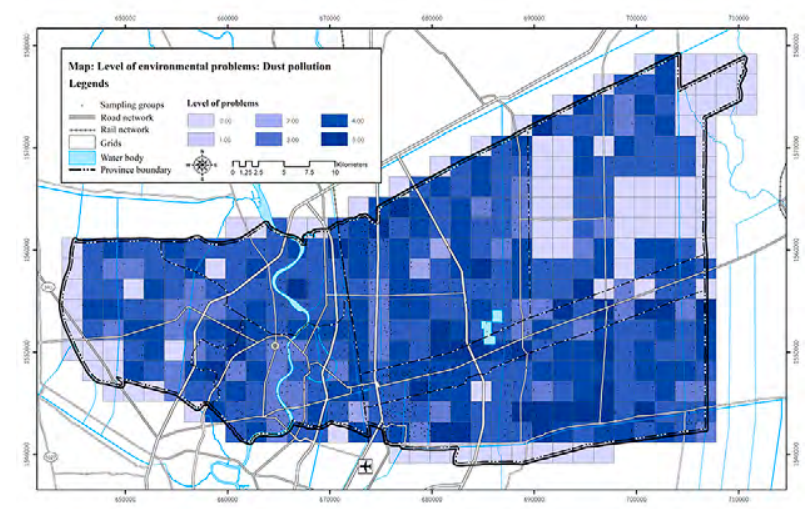

E. Dust pollution

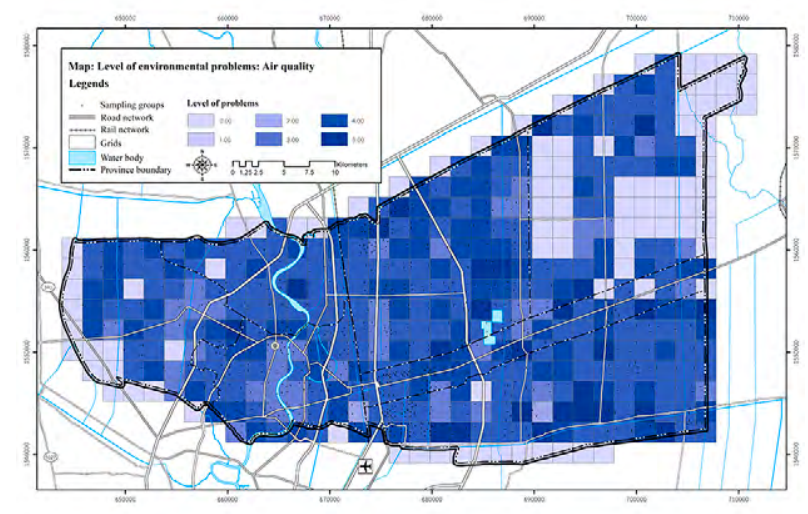

G. Air quality

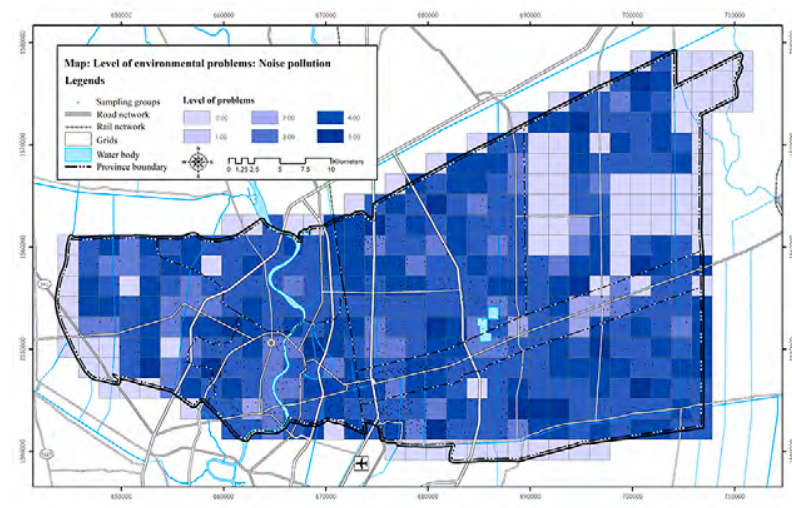

F. Noise pollution

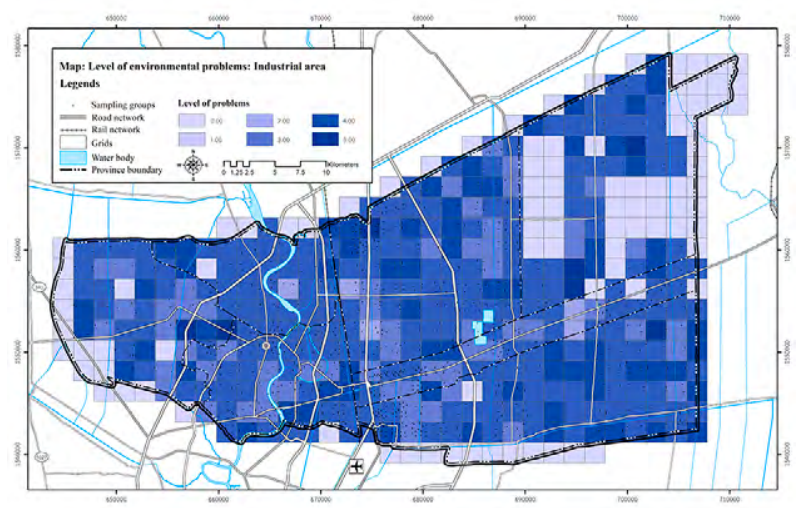

H. Industrial area

Figure 4. Average score of different built environment factor (continued from the previous page)

In terms of residents' perspective on physical aspects, the variables relating to the living conditions of the neighbourhoods and the community would be selected to represent the satisfaction of the neighbourhood environment. The more liveable condition will present the tend to be satisfied with the current environment. However, in terms of the environment, the reflection of respondents showed a negative direction with lower scores, especially, on issues of dust and air pollution that is being faced by other areas aside Pathumthani province. This problem is already at the macro level and it is facing everyone which necessitates collective search for solution to ameliorate the situations. However, in terms of policy, it can be seen that there is no effective measurement in place to tackle this current problem. The score value for this factor could obviously be seen to be the lowest when compared to other factors.

\section{Health outcomes}

Considering, health status that the number of congenital disease data were collected from questionnaires, asked whether there is an underlying disease or not. It was found that the most common diseases found in the study area were hypertension, allergy, diabetes, heart disease, dyslipidemia, pneumonia, obesity, cancer, and neuropathy. Therefore, environmental quality assessment based on the interviewers provide the direction with self-reported indicators both health condition and perceived neighborhood quality. Health outcomes was considered in term of the diseases. The analysis was performed by using explanatory variable of health status representing on the basis of number of chronic diseases (Figure 5).

\section{The classifications of relationship between built environment and health effects}

The impact of cities on health is both direct and indirect, and most of urban health problems are caused by interrelated factors. In the dimension of urban development, the influencing factors should cover multidimension of urban aspects. However, it must consider all urban element in term of the physical, social and economic environment of urban areas which comprise of the way of living and behaviour of people. Analysis of the composition of perception on built environment factors produced from data collection based on in depth interview and factor scores provide a quantification tool to summarize health information based on an influencing from a number of variables in a manageable and meaningful form of association of urban components. On classifying different built environment factors in our case study, the result of clustering on the relationship of built environment 


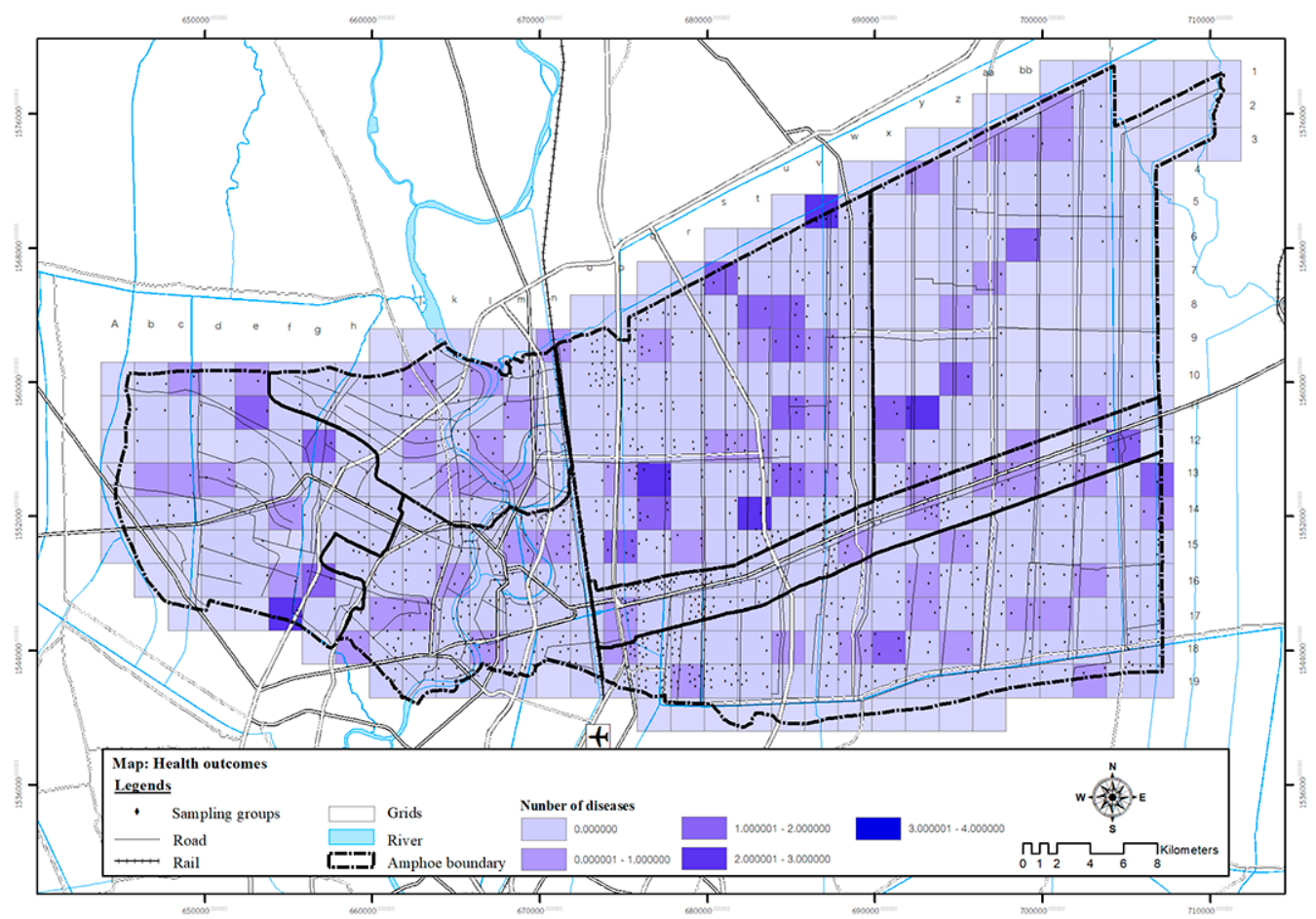

Figure 5. Clustering of Health outcomes

that affects the residents' health condition are shown in Table 1 and Table 2. The cumulative percentage (cumulative \%) presents that the factors obtained after the analysis among all 3 group of factors were able to explain the total variability of the variables 65.122 percent. The spatial visualization of different clusters in the study area can be demonstrated in Figure 6. mental quality score which reflected by their attitude. Furthermore, it was found that most of the residents demonstrate their moderate satisfaction on neighbourhood environment which might be positively influenced by the liveability of physical features of transportation, housing, industrial areas, and infrastructure systems.

Table 1. Eigenvalues from successive extractions of principal components from built environment factors

\begin{tabular}{|l|c|c|c|}
\hline \multirow{2}{*}{ Factors } & \multicolumn{3}{|c|}{ Clusters } \\
\cline { 2 - 4 } & $\mathbf{1}$ & $\mathbf{2}$ & $\mathbf{3}$ \\
\hline Dust pollution & 0.806 & -0.104 & -0.211 \\
\hline Air Quality & 0.760 & -0.148 & -0.369 \\
\hline Noise & 0.717 & 0.261 & 0.345 \\
\hline Water Quality & 0.667 & 0.010 & -0.168 \\
\hline Housing & -0.060 & 0.813 & -0.045 \\
\hline Infrastructures & -0.098 & 0.781 & -0.048 \\
\hline Transportation & 0.094 & -0.447 & 0.673 \\
\hline Industrial Areas & 0.443 & 0.381 & 0.551 \\
\hline
\end{tabular}

Group 1: The main concern of residents in this group are in relation with environment pollution which comprises of water quality, noise pollution, air quality and dust pollution. The level of the health condition of most of the residents demonstrates the good level which can be the best characterized their satisfaction on households' local environment. This is due to the value of moderate to high level of the environ-
Group 2: The characteristic of this group is characterized as a moderate level of satisfaction on their environmental quality based on the attitude of the people who live in the area. In terms of the satisfaction of the urban infrastructure (transportation, utility and facility) and urban environment (water quality, noise pollution, air quality and dust pollution), most of household and community focus heavily on 
Table 2. Classification of different the built environmental factors and health impacts

\begin{tabular}{|l|c|c|c|c|c|c|c|}
\hline \multirow{2}{*}{ Factors } & \multicolumn{7}{|c|}{ Clusters } \\
\cline { 2 - 9 } & \multicolumn{2}{|c|}{$\mathbf{1}$} & \multicolumn{2}{c|}{$\mathbf{2}$} & \multicolumn{2}{c|}{$\mathbf{3}$} & P-value \\
\hline Transportation & 2.95 & Medium & 2.73 & Medium & 2.74 & Medium & $0.000^{*}$ \\
\hline Water quality & 3.28 & Medium & 2.57 & Medium & 2.72 & Medium & $0.000^{*}$ \\
\hline Noise pollution & 3.33 & Medium & 2.81 & Medium & 2.16 & Medium & $0.000^{*}$ \\
\hline Housing & 2.76 & Medium & 2.02 & Medium & 2.89 & Medium & $0.000^{*}$ \\
\hline Industrial areas & 3.28 & Medium & 3.41 & High & 2.54 & Medium & $0.000^{*}$ \\
\hline Infrastructures & 2.73 & Medium & 1.94 & Medium & 2.83 & Medium & $0.000^{*}$ \\
\hline Air Quality & 3.16 & Medium & 1.80 & Medium & 2.58 & Medium & $0.000^{*}$ \\
\hline Dust pollution & 3.27 & Medium & 1.73 & Medium & 2.27 & Medium & $0.000^{*}$ \\
\hline Health condition & 3.71 & High & 3.65 & High & 3.63 & High & 0.063 \\
\hline
\end{tabular}

*Note: statistics significance at 0.050 with 1,000 data sets. The highlight on different built environment aspects represents the explanatory factor of the specific cluster.

Remark: Built environment and health aspect are classified into 3 ranges which consist of: High level (3.34-5.00 score), Medium level (1.67-3.33 score) and Low level (0.00-1.66 score).

community-level aspects of infrastructure, particularly on improvement in local accessibility and access to local services. Although, inadequate information to capture the variation in environmental quality that is present across settlement type in the study area, this cluster presents the high satisfaction score on industrial area which might be due to the residential allocation of this cluster is located within the development of a green environmental protection industry. In terms of health in consideration to congenital disease dimension, the health means was at a moderate level. As for the health condition, it is found that average score of the satisfaction on their own physical health of this group are at the high level.

Group 3: This group is classified by the satisfaction level of transportation and industrial areas factor. The influence of man-made structures on environmental preference also leads to the environmental problem which related to congestion problem with proportion and scale of imbalance space utilization together with degradation of urban environment quality. Although, the perception on quality of urban environmental, particularly around industrial area presents the moderate level, the scoring shows clear pattern of

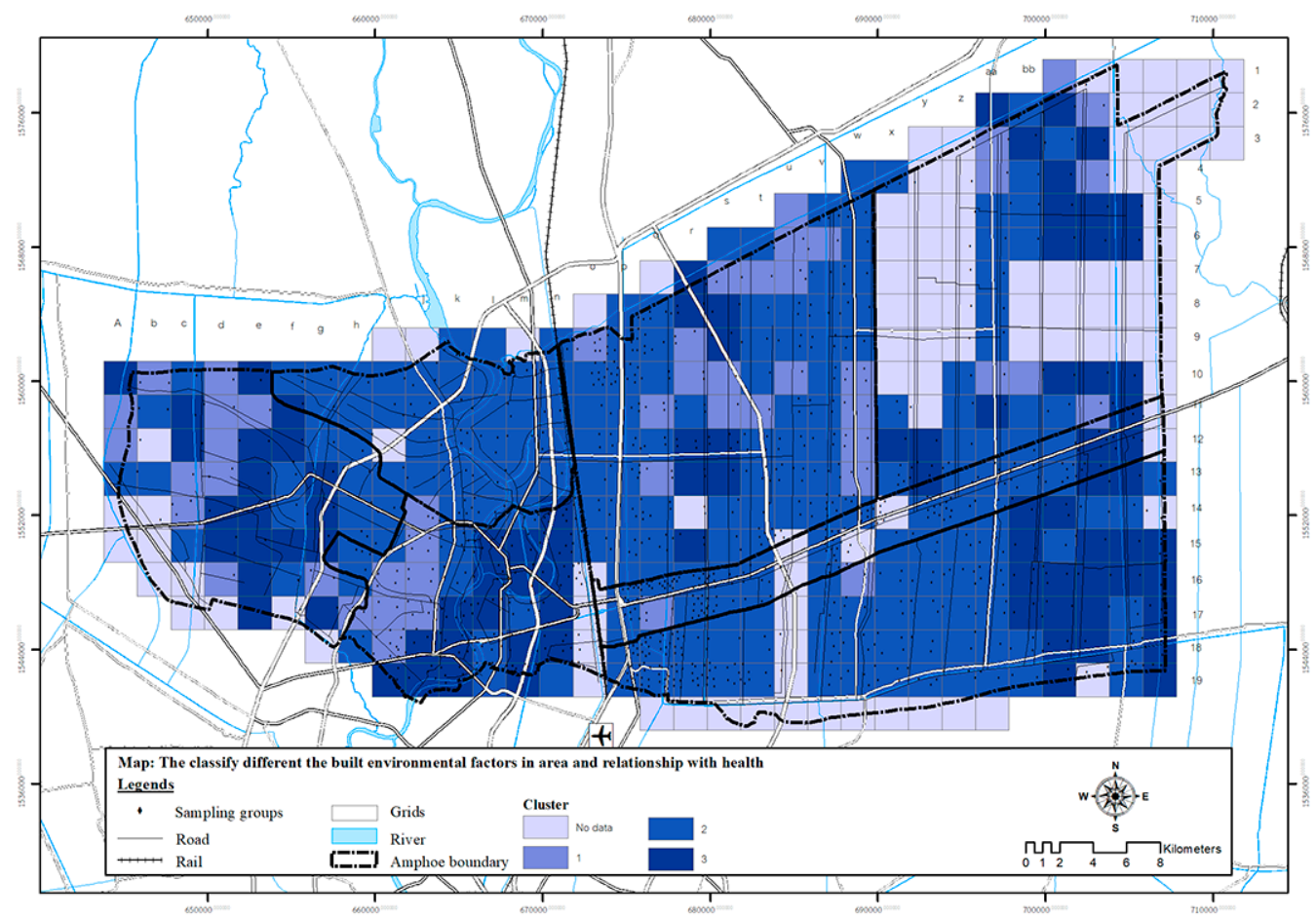

Figure 6. Clustering of different built environmental factors in the study area 
environmental impact (water quality, noise and dust pollution) around their neighbourhoods that resulted to low quality living conditions. On another hand, there may be a diverse range of housing choice that is affordable around this cluster's community with well- linked to public transport, walking and cycling infrastructure (moderate level of satisfaction). For health factor, average health score is found to be good, however the overall perspective of environmental quality still not led them to high quality of life.

\section{Discussion}

It is interesting to derive residents' opinion of their satisfaction on built environment nearby neighbourhoods (noise pollution, water pollution, dust pollution, air quality, industrial area, housing, infrastructures and transportation) together with their health condition (number of congenital diseases). The satisfaction assessment based on Likert scale was input for clustering their spatial classification. The three group of built environments that affects the residents' health condition can be categorized into three major domains as described in the following detail (Table 2).

\section{Group 1: Perception of Pathumthani residents toward urban environment}

The first group of residents demonstrated their high level of preferences in all built environment factors and their health status compared to others. However, the water quality (3.28), noise pollution (3.33), air quality (3.16) and dust pollution (3.27) are the key concerned for this group and represented as major environmental stressors in urban areas. The preferences among local people are always overlooked in the local plan to reflect the status quo of urban environment condition. Furthermore, it was found that most of the environmental problems in Pathumthani province are due to the impacts of rapid development with the sprawling of suburban. This is consistent with the study of Frank and Engelke (2005) stated that urban density is the main feature of built environment and relates to the level of air quality. Additionally, with the rapidly expanding across several communities with hundreds of housing projects in Pathumthani province as well as industries are the major source of wastewater. Some of the water pollution arising from those sources has been treated to the effluent quality standards, while some have not been treated before draining into the sewer system. In addition, air quality and noise problems in Pathumthani province have been discovered to be as a result of the presence of industrial city and continuous expansion of Bangkok; resulting into a large number of establishments. This also leads to the problem of noise pollution in Pathumthani province which can be classified into 2 categories according to the source of the pollution; industrial areas and traffic congestion. The current urban planning and management is necessary to cope with the environmental degradation, particularly on carbon footprint from urban development by prioritising climate-friendly measurement.

\section{Group 2: Perception of Pathumthani residents toward urban facilities and services}

The opinion of the second group shows their concerns related to social aspects of city life, in particular to level of satisfaction, experiences, and perception of their everyday environment on living (2.02) and utilizing urban infrastructures (1.94). The reflection of residents (as main users) in term of quality of living and community well-being can provide an valuable information to address these complex urban challenges, and particularly for improving plan for 'area-based' interventions. It was found that housing quality is one of the main factors associated with everyday life at individual level. Therefore, improvements in housing quality is highly recommended since it is associated with positive general health, mental health, asthma, and mortality outcomes (Frank \& Engelke, 2005). It is a fact that housing factors are related with mental and physical health impacts, e.g., air quality, infestation, noise, lighting, housing tenure and design (Macdonald \& Thompson, 2003). This assertion is in consistent with the study results from Gibsona et al. (2011) and Sandel et al. (2018), which was found strong evidence on characterizing housing's relationship to health. The characteristics of the living environment that has different conditions according to the socioeconomic context (Iamtrakul \& Chayphong, 2021). Thus, the enhancement on housing stability, quality, safety, and affordability will definitely address their effects on health outcomes (physical and social characteristics of neighborhoods).

\section{Group 3: Perception of Pathumthani residents toward urban accessibility and attractions}

It is important to note that the transportation problems (2.74) and industrial areas (2.54) influence on this group of residents' preferences. The perception of residents concerning their health and well-being due to node of intensive industrial activities and its density relates to the level of traffic. The suburban development of Pathumthani showed significant associations and the urban atmosphere around industrial 
that has tendency to exacerbate exposure to harmful emission, especially heavy vehicles mixed and traffic congestion. Concentration of industrial activities, notably those of large-scale petrochemical, power generation and heavy industrialization creates environmental pressures. Combined with all of the economic activities within that area has potential adverse effects on the health of local communities through their occupational and residential roles (World Health Organization, 2004). This is due to the possibility of di- rect exposure to PM2.5 and NOX from industries and these are associated with decrease lung function (Bergstra, 2018). The environmental problem and other situations in the province were related to several sites of industrial activities with low efficient network of transportations. Thus, the integrated of friendly transportation system such as rail-based transport must be recommended to be consistent with allocation of urban attractions while increasing the accessibility and connectedness of non-motorization modes.

\section{Conclusion}

The aim of this study is to identify the relationship between built environment and urban health through perspective of residents. Data collection was sampling from Pathumthani province to understand people's preferences for built environment characteristic. The number of questionnaires was input into each grid in order to cover the sample in spatial area. We then analyzed the association on the basis of satisfaction of built environment factors (transportation, water quality, noise pollution, housing, industrial areas, infrastructures, air quality, dust pollution) which is consistent with health condition of residents in the study area. By applying the cluster analysis, the results of the classification were made into 3 clusters. On considering the perception of Pathumthani residents toward its environmental quality through spatial cluster analysis, it was found that the factors relating to or affecting the health issues emanated from the condition of the built environment. There are several dimensions of environmental factors that are related to health issues reflected from preferences of residents. This study classified three group of perception of Pathumthani residents which comprises of urban environment, urban facilities and services and urban accessibility \& attractions. The results of assessment can represent as a promising tool to integrate local perception by asking their response towards different built environment aspects which is essential step of participatory planning approach. The evidence based on level of perception among residents in the target area could demonstrate how people perceive the quality of local government plan and services through their planning and management. It can be seen that built environment plays a key role in support of systematic environmental management, eco-industrial cities development and promotion, and development of friendly transportation network and logistics system. Thus, the quantification of individual level contextual differences based on perception of residents concerning health and well-being can provide useful information for improving built environments and measurement to improve community health behaviors as well. This is due to significant findings of association between health-related quality of life and urban context. Finally, consideration should be given to the people and communities for the capacity to access high quality of service and infrastructure to support the continuous growth of their city in term of basic infrastructure, sufficient utility system, and effectively provision.

\section{Acknowledgement}

The research was supported by a grant from Faculty of Architecture and Planning, Thammasat University under the project entitled "A Study on Influencing Factors of Healthy Neighborhood Development in Suburban Context of Pathumthani Province". This research unit is supported by the Faculty of Architecture and Planning Research Fund, Thammasat university. It is also supported partially by the Center of excellence in urban mobility research and innovation, Thammasat university. 


\section{References}

Barton, H., \& Grant, M. (2006). A health map for the local human habitat. Journal of the Royal Society for the Promotion of Public Health, 126(6), 252-261. DOI: $10.1177 / 1466424006070466$

Bergstra, A.D., Brunekreef, B., \& Burdorf, A. (2018). The effect of Industrialization-related air pollution on lung function and respiratory symptoms in school children. Environmental Health volume, 17, 30. DOI: 10.1186/s12940-018-0373-2

Borasi, G., Zardini, M., Campbell, M., Ellin, N, \& Gissen, D. (2012). Imperfect health: the medicalization of architecture, Montreal, Canada. Canadian Centre for Architecture.

Bureau of technical advisors. Situation of health and environment in Thailand http://www.anamai.moph.go.th/ewtadmin/ewt/advisor/main. php?filename $=$ env201 (15.10.2020).

Centers for Disease Control and Prevention. (2011). Impact of the built environment on health. CS216341.

Christian, H., Giles-Corti, B., Knuiman, M., Timperio, A., \& Foster, S. (2011). The influence of the built environment, social environment and health behaviors on body mass index. Results from RESIDE. Preventive medicine, 53(1-2), 57-60. DOI: 10.1016/j.ypmed.2011.05.004

Collaborative on Health and the Environment https://www.healthandenvironment.org/environmental-health/environmental-risks/built-environment (20.10.2020).

Durand, C. P., Tang, X., Gabriel, K. P., Sener, I. N., Oluyomi, A. O., Knell, G., Porter, A.K., Hoelscher, D.M. \& Kohl III, H. W. (2016). The association of trip distance with walking to reach public transit: Data from the California Household Travel Survey. Journal of transport \& health, 3(2), 154-160. DOI: 10.1016/j.jth.2015.08.007

Ebmeier, S. (2012). SPICe Briefing 12/26: Climate change and health in Scotland. Edinburgh: Scottish Parliamentary Information Centre.

Ewing, R., \& Handy, S. (2009). Measuring the unmeasurable: Urban design qualities related to walkability. Journal of Urban design, 14(1), 65-84.

Frank, L. D., \& Engelke, P. (2005). Multiple impacts of the built environment on public health: walkable places and the exposure to air pollution. International regional science review, 28(2), 193-216. DOI: $10.1177 / 0160017604273853$

Frumkin, H., Frank, L., \& Jackson, R. (2004). Urban Sprawl and Public Health. Designing, Planning and Building for Healthy Communities, Washington DC: Island Press.
Gibsona, M., Petticrewb, M., Bambrac, C., Sowdend, AJ., Wrightd, KE., \& Whiteheade, M. (2011). Housing and health inequalities: A synthesis of systematic reviews of interventions aimed at different pathways linking housing and health. Health \& Place, 17, 175-184. DOI: 10.1016/j.healthplace.2010.09.011

Grant, M., Barton, H., Coghill, N., \& Bird, C. (2009). Evidence Review on the Spatial Determinants of Health in Urban Settings. Bonn: WHO European Centre for Environment and Health.

Iamtrakul, P. \& Chayphong, S. (2021). Healthy Aging in Home Environment Exposures. GMSARN International Journal, 15, 175-184.

Iamtrakul, P., Raungratanaamporn, I. \& Klaylee, J. (2018). The impact of urban development on social capital in urban fringe area of Bangkok, Thailand. Lowland Technology International, 20(3), 331-340.

Macdonald, C., \& Thompson, H. (2003). Housing improvement and health: Research findings. In: Health Impact Assessment of Housing Improvements: a guide. Glasgow: Scottish Health and Inequalities Impact Assessment Network.

McLeroy, K. R., Bibeau, D., Steckler, A., \& Glanz, K. (1988). An ecological perspective on health promotion programs. Health Education Quarterly, 15(4), 351-377. DOI: 10.1177/109019818801500401

Nathan, A., Villanueva, K., Rozek, J., Davern, M., Gunn, L., Trapp, G., Boulangé, C., \& Christian, H. (2018). The Role of the Built Environment on Health Across the Life Course: A Call for CollaborACTION. American journal of health promotion, 32(6), 14601468. https://doi.org/10.1177/0890117118779463a

Office of Knowledge Management and Development http://www.okmd.or.th/okmd-opportunity/urbanization/256/ (12.10.2020).

Office of the Surgeon General. (2015). Step It Up! The Surgeon General's Call to Action to Promote Walking and Walkable Communities, US Department of Health and Human Services.

Pathumthani Provincial Public Health Office. http://203.157.108.3/pathum/index.php (12.10.2020).

Perdue, W. C., Stone, L. A., \& Gostin, L. O. (2003). The built environment and its relationship to the public's health: the legal framework. American journal of public health, 93(9), 1390-1394. DOI: 10.2105/ ajph.93.9.1390

Pinter-Wollman, N., Jelic, A., \& Wells, N. M. (2018). The impact of the built environment on health behaviours and disease transmission in social systems. Philosophical Transactions of the Royal Society B: Biological Sciences, 373(1753). DOI: 10.1098/ rstb.2017.0245 
Sandel, M., Sheward, R., Ettinger de Cuba, S., Coleman, S.M., Frank, D.A., Chilton, M., Black, M., Heeren, T., Pasquariello, J., Casey, P., Ochoa, E., \& Cutts. D. (2018). Unstable Housing and Caregiver and Child Health in Renter Families, Pediatrics, 141(2), 1-12. DOI: 10.1542/peds.2017-2199.

Sherrington, C. S. (1961). The integrative action of the nervous system. New Haven: Yale University Press.

Stokols, D. (1996). Translating social ecological theory into guidelines for community health promotion. American Journal of Health Promotion, 10(4), 282-298. DOI: 10.4278/0890-1171-10.4.282.

Sundquist, K., Eriksson, U., Mezuk, B., \& Ohlsson, H. (2015) Neighborhood walkability, deprivation and incidence of type 2 diabetes: a population-based study on 512,061 Swedish adults. Health Place, 31, 24-30. DOI: 10.1016/j.healthplace.2014.10.011

Sustainable Development Commission. (2008). Health, place and nature. How outdoor environments influence health and well-being: a knowledge base. London: Sustainable Development Commission.

Taro, Y. (1967). Statistics, An Introductory Analysis, 2nd Ed., New York: Harper and Row.
Teo, T.L., Coleman, H.M., \& Khan, S.J. (2015). Chemical contaminants in swimming pools: occurrence, implications and control. Environment International, 76, 16-31. DOI: 10.1016/j.envint.2014.11.012

Tsekleved, E., \& Cooper, R.F.D. (2017). Emerging Trends and the Way Forward in Design in Healthcare: An Expert's Perspective. The Design Journal, 20(1), S2258-S2272. DOI: 10.1080/14606925.2017.1352742

Villanueva, K., Pereira, G., \& Knuiman, M, Bull, F., Wood, L., Christian, H., Foster, S., Boruff, B.J., Beesley, B., Hickey, S. \& Joyce, S. (2013). The impact of the built environment on health across the life course: design of a cross-sectional data linkage study. BMJ Open, 3(1), 1-11. DOI: 10.1136/bmjopen-2012-002482

WHO. (1946). Preamble to the Constitution of WHO as adopted by the International Health Conference. World Health Organization. (2004). The Physical School Environment: An Essential Component of a Health-Promoting School.

World urbanization prospects. (2018). The 2018 Revision of World Urbanization Prospects. 